\title{
Low Temperature Static Behavior of the Two-Dimensional quantum Easy-Axis Heisenberg Model
}

\author{
S. A. Leonel* and A. S. T. Pires ${ }^{+}$ \\ ${ }^{*}$ Departamento de Física, ICE Universidade Federal de Juiz de Fora, \\ Juiz de Fora, CEP 36036-330, MG, Brazil \\ ${ }^{+}$Departamento de Física, ICEx, Universidade Federal de Minas Gerais \\ Belo Horizonte, CP 702, CEP 30123-970, MG, Brazil
}

Received 2 January, 2000

\begin{abstract}
We use the self-consistent harmonic approximation (SCHA) to study static properties of the twodimensional quantum Heisenberg model with easy-axis anisotropy. We calculate the critical temperature as a function of the spin value, and compare with classical results. Specifically, we compare how the ratio of critical temperatures varies as a function of the spin $\mathrm{S}$ in the quantum and classical cases, for a fixed anisotropy parameter. We see that, for values of spin near $5 / 2$, the classical result approximates to the quantum results and the classical calculation is justified. We have also studied the behavior of the magnetization for very small anisotropies. We have shown that our magnetization curves do not present a plateau in the limit of very small anisotropies, as predicted by the real-space renormalization-group calculations.
\end{abstract}

\section{Introduction}

Low-dimensional magnets have been extensively investigated by many theorists and experimentalists in the last three decades. More recently, the interest on the properties of two-dimensional (2D) Heisenberg magnets has been greatly revived since the discovery of high- $\mathrm{T}_{c}$ superconductivity: it is now well known[1] that the undoped, insulating $\mathrm{La}_{2} \mathrm{CuO}_{4}$ has a quasitwo-dimensional antiferromagnetic behavior. However, most quasi-two-dimensional magnetic real materials exhibit some kind of anisotropy: the anisotropic properties often arise not so much from an anisotropy in the interaction mechanism (which can be wholly isotropic) but from other sources, such as the presence of a crystal field that couples the spins to a certain direction in the crystal. Then, at least from a theoretical point of view, a large amount of magnetic materials fits (under certain circunstances like temperature range) into one of the two groups: easy-plane or easy-axis models. Easy-plane 2D magnets have deserved a lot of attention due to their possibility of showing the topological Kosterlitz-Thouless phase transition.[2] The interest devoted to easy-axis magnetic systems has been considerably smaller.

In a recent paper[3], we have studied the static and dynamical behavior of the two-dimensional classical easy-axis Heisenberg model. In the refered paper we have used a classical treatment and our analytical results, for both static properties and dynamic correlation functions, were compared to numerical sim- ulation data combining cluster-Monte Carlo algorithms and Spin Dynamics. The comparison allowed us to conclude that below the transition temperature, where the SCHA is valid, spin waves are responsible for all the relevant features observed in the numerical simulation data: topological excitations did not seem to contribute appreciably.

It must be emphasized that, although we shall be concerned only with magnetic systems in this paper, many of the magnetic Hamiltonians also allow for an interpretation other then a magnetic one. Most physical problems concerning mutually interacting elements that form a spatial array can be mapped into a magnetic Hamiltonian by describing it within a pseudo spin formalism. The advantadge of studying a general physical problem in its magnetic form is clearly that in magnetism several experimental techniques are available to study the fundamental properties of a system.[4]

The analysis of the general Ising-Heisenberg model is of interest because, from the experimental point of view, the presence of some degree of anisotropy in the interaction is to be expected in nearly all cases.

Here we consider the two dimensional Heisenberg ferromagnet with easy-axis exchange anisotropy

$$
H=-J \sum_{\mathbf{n}, \mathbf{a}} \mathbf{S}_{\mathbf{n}} \cdot \mathbf{S}_{\mathbf{n}+\mathbf{a}}-K \sum_{\mathbf{n}, \mathbf{a}} S_{\mathbf{n}}^{z} S_{\mathbf{n}+\mathbf{a}}^{z}
$$

where the summations run over all distinct pairs of spin sites $\mathbf{n}$ and its nearest neighbors a. As the anisotropy parameter $K$ ranges from 0 to $\infty$, we go 
from the isotropic Heisenberg model to an Ising like model in which the spins tend to be confined along the $\pm z$-direction.

In this paper we consider the contribution of spin waves, using a self-consistent harmonic approximation theory (SCHA). As is well known, the SCHA is a reasonable approximation to calculate the transition temperature and low-temperature properties of a system but it is of limited value in estimating critical properties. Therefore, in our work, we did not attempt to do any calculation for critical exponents and related aspects of a phase transition.

We use a quantum treatment and compare our result with the classical one in order to see when the classical calculation is justified.

We give our quantum results for the critical temperature as a function of the anisotropy parameter $K / J$, for a fixed value of the spin, and for small and large anisotropies. We find that, for large anisotropies, the critical temperature are given as $T_{c} \approx 2.00 \mathrm{~K}$, to be compared with the $2 \mathrm{D}$ single-component Onsager result for the Ising model value, $T_{c} \approx 2.27 \mathrm{~K}$.

In the limit of small anisotropies, we find that contrary to the real space renormalization-group calculation[5], the magnetization curves does not exhibit a plateau as a function of temperature, wich is in agreement with the calculation of Reinehr and Figueiredo[6].

\section{Self-Consistent Approximation}

Since its original derivation by Bloch,[7] the self consistent harmonic approximation has been found to account for the low temperature dependence of various properties of several magnetic insulators, which seem to be fairly well-described by the Heisenberg model. $[8,9,10]$ Its usefulness stems mainly from the way it takes into account a substantial part of the interactions among spin waves, being characterized by simple temperaturedependent renormalization factors for the unperturbed spin wave energy.

We start by writing the spin components using the Dyson-Maleev representation of spin operators

$$
\begin{aligned}
S_{\mathbf{n}}^{x} & =\frac{1}{2}\left[\sqrt{2 S}\left(a_{\mathbf{n}}^{\dagger}+a_{\mathbf{n}}\right)\right]-\frac{1}{\sqrt{8 S}} a_{\mathbf{n}}^{\dagger} a_{\mathbf{n}} a_{\mathbf{n}} \\
S_{\mathbf{n}}^{y} & =\frac{1}{2 i}\left[\sqrt{2 S}\left(a_{\mathbf{n}}^{\dagger}-a_{\mathbf{n}}\right)\right]-\frac{1}{\sqrt{8 S}} a_{\mathbf{n}}^{\dagger} a_{\mathbf{n}} a_{\mathbf{n}} \\
S_{\mathbf{n}}^{z} & =S-a_{\mathbf{n}}^{\dagger} a_{\mathbf{n}}
\end{aligned}
$$

where $a_{\mathbf{n}}^{\dagger}$ and $a_{\mathbf{n}}$ are the Bose spin operators on site n. The harmonic spin wave Hamiltonian obtained from
(1) is given by

$$
H_{0}=\sum_{\mathbf{q}} \omega_{\mathbf{q}} a^{\dagger} a^{a} \mathbf{q}
$$

where $a_{\mathbf{q}}^{\dagger}$ and $a_{\mathbf{q}}$ are the Fourier transforms of $a_{\mathbf{n}}^{\dagger}$ and $a_{\mathbf{n}}$ respectively, and

$$
\omega_{\mathbf{q}}=4 J S[1-\gamma(\mathbf{q})]+4 K S
$$

with $\gamma(\mathbf{q})=\frac{1}{2}\left[\cos q_{x}+\cos q_{y}\right]$. The spin wave approximation will be reasonable when $\left\langle a_{\mathbf{n}}^{\dagger} a_{\mathbf{n}}\right\rangle \ll S$, so it ought to be fairly good for anisotropies satisfying the relation $T \ll 4 K S^{2}$.

Now we simplify the general model by reducing Hamiltonian (1) to an effective harmonic problem with the effect of the anharmonicity embodied in temperature-dependent renormalized parameters. This means that the couplings of the model are replaced by quadratic interactions whose strength is then optimized. Details of this method may be found in the literature [7, 9] and here we give only an outline of those steps pertinent to our present calculation.

We assume as effective Hamiltonian the appropriate form for a noninteracting gas of Bose excitations

$$
\tilde{H}_{0}=\sum_{\mathbf{q}} E_{\mathbf{q}} a_{\mathbf{q}}^{\dagger} a_{\mathbf{q}} .
$$

The spin wave energy is obtained by a variational procedure based on the inequality for the free energy $F$

$$
F \leq \tilde{F}_{0}+\left\langle H-\tilde{H}_{0}\right\rangle_{0},
$$

where the brackets indicate the thermal average. Traces should be taken only over the physical states, that is, states with no more than $2 S$ spin deviations on a single site. The minimization of (5) with respect to $E_{\mathbf{q}}$ determines the spin wave energies. We obtain, following Rastelli et al,[9]

$$
\begin{aligned}
E_{\mathbf{q}}(T) & =4 J S(1-\gamma(\mathbf{q}))[1-\tilde{\beta}(T)+\tilde{\eta}(T)] \\
& +4 K S[1-\tilde{\beta}(T)-\gamma(\mathbf{q}) \tilde{\eta}(\mathbf{T})]
\end{aligned}
$$

where

$$
\begin{gathered}
\tilde{\beta}(T)=\frac{1}{N S} \sum_{\mathbf{q}} n_{q} \\
\tilde{\eta}(T)=\frac{1}{N S} \sum_{\mathbf{q}} \gamma(\mathbf{q}) n_{q}
\end{gathered}
$$

Here $n_{q}$ is the Bose distribuition

$$
n_{q}=\left(e^{E_{q}(T) / T}-1\right)^{-1}
$$

Eqs. (7), (8), and (9) are coupled equations which should be solved self consistently by an iterative method. These coupled equations have a double-valued 
solution below $T_{c}$ and no real solution above $T_{c}$ : this is the typical behavior for self consistent harmonic approximations and allows for easy determination of $T_{c}$. The lower branch (for $T<T_{c}$ ) has an unphysical temperature dependence and may be discarded as a spurious mathematical solution that is physically unstable.

\section{Static Results}

In Fig. 1 the ratio between the quantum and classical calculation for the critical temperature, for two values of anisotropy $K / J=1.0$ and $K / J=10.0$, are given as a function of the spin. We see that when the value of the spin increases, the ratio between quantum and classical results approximate to one. This behavior is expected for the domain of validity of the classical approximation.

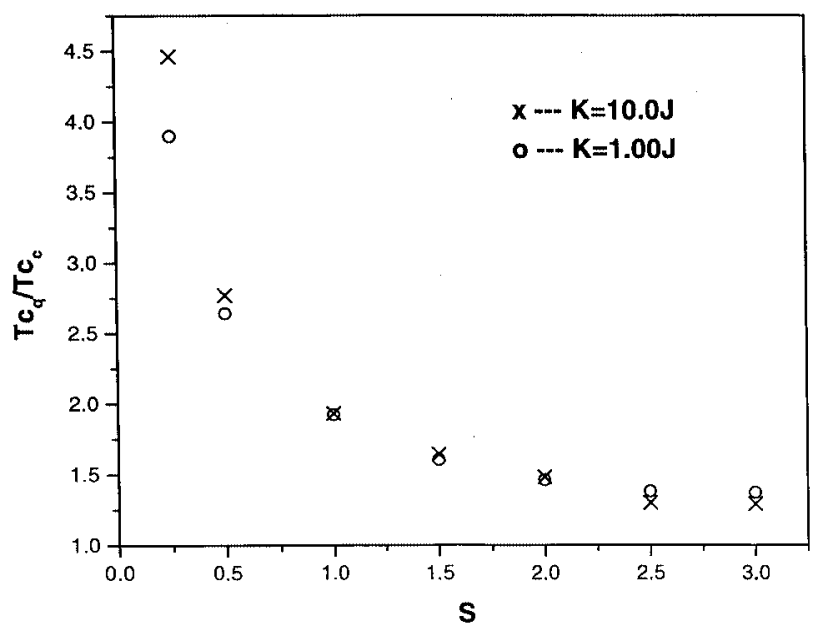

Figure 1. Ratio between quantum critical temperature and classical critical temperature as a function of spin $S$, for two value of anisotropies, $K=10.0 \mathrm{~J}$ and $K=1.0 \mathrm{~J}$.

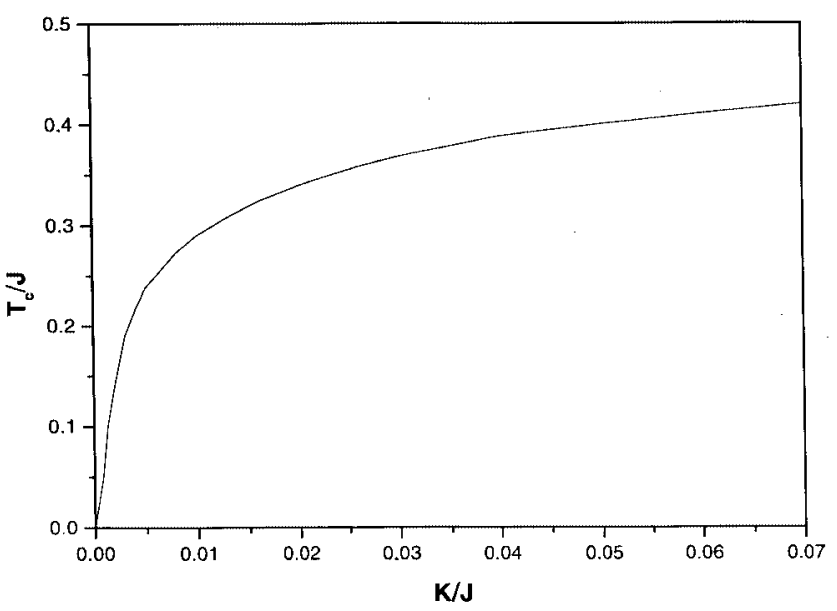

Figure 2. Reduced critical temperature $T_{c} / J$ as a function of anisotropy parameter $K / J$,for small anisotropies and $S=1 / 2$.

In Fig. 2 we show the critical temperature behavior as a function of the anisotropy parameter $K / J$, for small anisotropies and spin $S=1 / 2$. We see when $K$ tends to zero, the hamiltonian (1) tends to the Heisenberg isotropic model and the critical temperature tends to zero, as expected.

In Fig. 3 we give the critical temperature as a function of the anisotropy parameter $K / J$, for $K / J>1.0$ and a constant value of spin $S=1$. Notice that, as $K$ increases, the dependence of $T_{c}$ on $K / J$ becomes linear. For $K / J \gg 1$, we recover a continuous spin Ising Hamiltonian: Eq. (1) can be approximated as $H \approx J(1+K / J) S_{\mathbf{n}}^{z} S_{\mathbf{n}+\mathbf{a}}^{z}=\tilde{K} S_{\mathbf{n}}^{z} S_{\mathbf{n}+\mathbf{a}}^{z}$. Fig. 3 shows that, for $K / J>1.0$, the results follow a straight line with slope $\approx 2.0$. For these anisotropies, $T_{c} \approx 2.0 \mathrm{~K}$ wich is near to the $2 \mathrm{D}$ single-component Ising model value, $T_{c} \approx 2.27 K$.

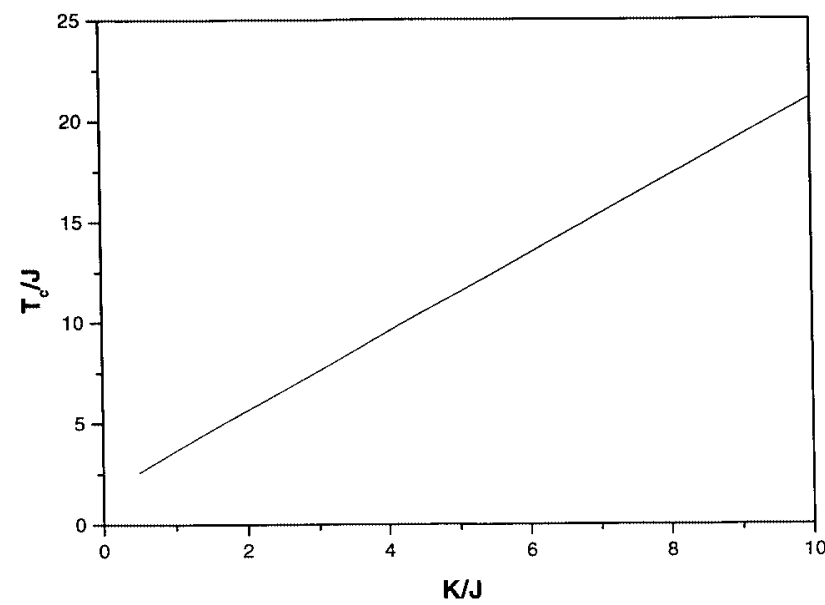

Figure 3. Reduced critical temperature $T_{c} / J$ as a function of the anisotropy parameter K for $S=1.0$.

The reduced spontaneous magnetization along the $z$-axis is given by

$$
\frac{M_{z}(T)}{M_{z}(0)}=1-\tilde{\beta}(T)
$$

In Fig. 4 we present results obtained from eq.(11) for small anisotropies, that is, $K / J=0.001, K / J=$ $0.01, K / J=0.07, K / J=1.0$, and the spin $S=1 / 2$. The magnetization calculated using this equation drops discontinously to zero at a temperature that we take as $T_{c}$. 


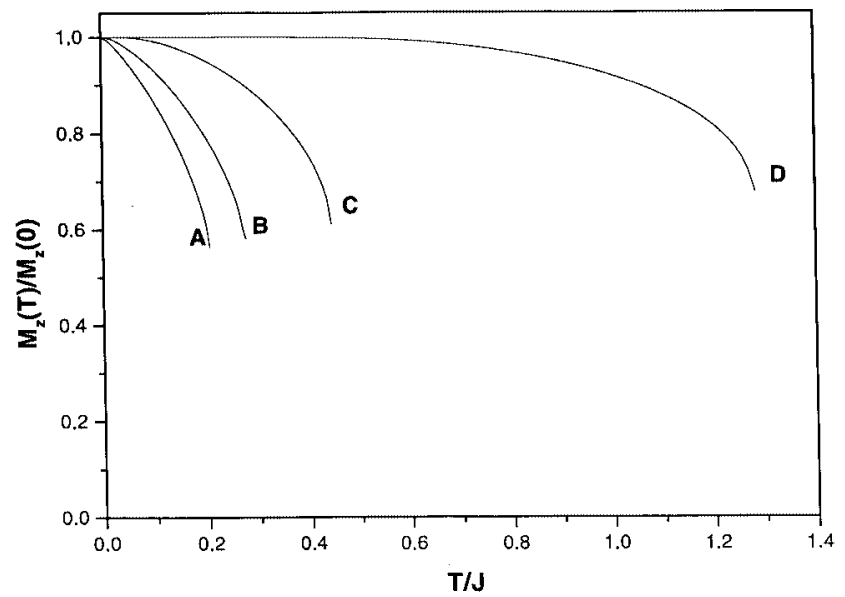

Figure 4. Reduced magnetization as a function of temperature for small anisotropies and $S=1 / 2$. Curve A correspond to $K=0.001 J$, curve B to $K=0.01 J$, curve C to $K=0.07 J$ and curve D to $K=1.0 J$.

In two recent papers $[5,6]$ the authors have calculated the magnetization as a function of temperature for this model with spin value $S=1 / 2$. In the first [5], the author have applied the real space renormalization-group and have show that, in the limit of small anisotropies, the magnetization curves exhibit a plateau as a function of temperature. In the second [6], where the authors have applid the formalism of Green's functions, they have shown that, in limit of small anisotropies, the magnetization curves did not exhibit a plateau as a function of temperature. In our calculation, the magnetization curves for small anisotropies does not exib a plateau, as Fig. 4 shows. Our calculations is then in agreement with the calculation of Reinehr and Figueredo[6].

\section{Conclusions}

We have applied a self-consistent harmonic approximation to the quantum easy-axis model, obtaining the critical temperature and magnetization curves. We have demonstrated that the quantum and classical results for the critical temperatures are close for values of the spin greater then one. Thus, we see that the classical approximation, for values of spin grater than one, is reasonable and the quantum effects are appreciable only for small values of the spin. In the limit of large anisotropies, that is $K / J>>1$, we get an Ising like model in which the spins tend to be confined along $\pm z$ direction. We find, in this limit, $T_{c} \approx 2.00 \mathrm{~K}$ which is near $T_{c} \approx 2.27 \mathrm{~K}$ for the $2 \mathrm{D}$ single-component Ising model.

We have also shown that the magnetization curves did not exhibit a plateau for small values of the anisotropy. This behavior is in agreement with that observed in the Green's function calculations and is different from that observed in the real-space renormalization calculations where a plateau appears.

\section{Acknowledgements}

We wish to thank Prof. Flávio I. Takakura for valuable discussion about this work.

The authors gratefully acknowledge the support of Fundação de Amparo à Pesquisa do Estado de Minas Gerais (FAPEMIG), Fundação Coordenação de Aperfeiçoamento de Pessoal de Nível Superior (CAPES) and Conselho Nacional de Desenvolvimento Científico e Tecnológico $(\mathrm{CNPq})$.

\section{References}

[1] Low Dimensional Conductors and Superconductors, edited by D. Jerome and L.G. Caron, NATO Advanced Studies Institute, Series B, Vol. 155 (Plenum, New York, 1987).

[2] J.M Kosterlitz and D.J. Thouless, J. Phys. C6, 1181 (1973); V.L. Berezinskii, Zh. Eksp. Teor. Fiz. 61, 1144 (1971) (Sov.Phys.-JETP).

[3] M.E. Gouvêa, G.M. Wysin, S.A. Leonel, A.S.T. Pires, T. Kamppeter and F.G. Mertens, Phys. Rev.B 59, 6229 (1999).

[4] L.J. de Jongh, ed. Magnetic Properties of Layered Transition Metal Compounds (Kluwer, Dordrecht, 1989).

[5] A. Chame, J. Phys. Condens. Matter 3, 9115 (1991).

[6] E.E. Reinehr and W. Figueiredo, Phys. Rev. B 52, 310 (1995).

[7] M. Bloch, J. Appl. Phys. 34, 1151 (1963).

[8] P.D. Loly, J. Phys. C 4, 1365 (1971).

[9] E. Rastelli, A. Tassi, L. Reatto, J. Phys. C 7, 1735 (1974).

[10] D.A. Poling, R.H. Parmenter, Phys. Rev B 16, 3240 (1977). 